\title{
SUPERNOVA REMNANTS
}

\author{
S. VAN DEN BERGH \\ David Dunlap Observatory, Richmond Hill, Ontario, Canada
}

\begin{abstract}
The present structure and recent changes in Cas A are discussed. On the deepest available exposures the optical remnant of this supernova is seen to consist of an almost complete shell. The southern part of this shell is outlined by knots that have developed during the last decade. It is pointed out that moving nebulosity in which [O III] radiation is particularly strong is distributed differently from nebulosity in which the $[\mathrm{O} \mathrm{III}] /[\mathrm{S} \mathrm{II}]$ ratio is more nearly normal. Observation of the motions of individual knots suggests a model in which dense blobs of matter, which have a highly anomalies composition, become luminous as they plough through a stationary interstellar (or circumstellar) medium.
\end{abstract}

\section{Introduction}

Observational data on all known optical supernova remnants have recently been summarized in 'An Atlas of Galactic Supernova Remnants' by van den Bergh et al. (1973). A more detailed discussion of the supernovae of the second millennium A.D. (Lupus 1006, Crab 1054, Tycho 1572, Kepler 1604 and Cas A 1667) is given by van den Bergh (1973).

In the present paper I shall confine myself to a brief discussion of some observations of Cassiopeia A that have recently been obtained with the 200-in. telescope. The first observations of Cas A are described by Baade and Minkowski (1954). Subsequent discussions of the optical observations of this supernova are given by van den Bergh and Dodd (1970) (expansion), van den Bergh (1971a, b) (photometry, spectroscopy), Peimbert and van den Bergh (1971), Peimbert (1971) (composition), and by Searle (1971) (reddening).

\section{Structure of Cas $A$}

Figure 1 shows a 200-min exposure of Cas A obtained through an [S II] interference filter that transmit light in the range 6650-6830 $\AA$. This deep exposure shows the following:

(a) The optical remnant of Cas A forms an almost complete shell with inner and outer radii of $\sim 80^{\prime \prime}$ and $\sim 120^{\prime \prime}$ respectively.

(b) The structure of the bright and faint nebulosity differs. The brightest moving nebulosity consists of crisp knots, whereas the faintest detectable nebulosity has a much more diffuse character.

(c) Figure 2 shows the distribution of all moving nebulosity in which [S II] appeared brighter on a $200-\mathrm{min} \lambda \lambda 6650-6830$ (S II interference filter $+098-02$ ) exposure than did [O III] on a 120-min $\lambda \lambda 4700-5500(\mathrm{GG} 7+103 \mathrm{aJ})$ exposure. In Figure 2 hatching represents the faintest nebulosity which is only marginally visible on the 200 -min exposure.

[O III] emission seems to be entirely absent from the knots labelled $A, B$ and 


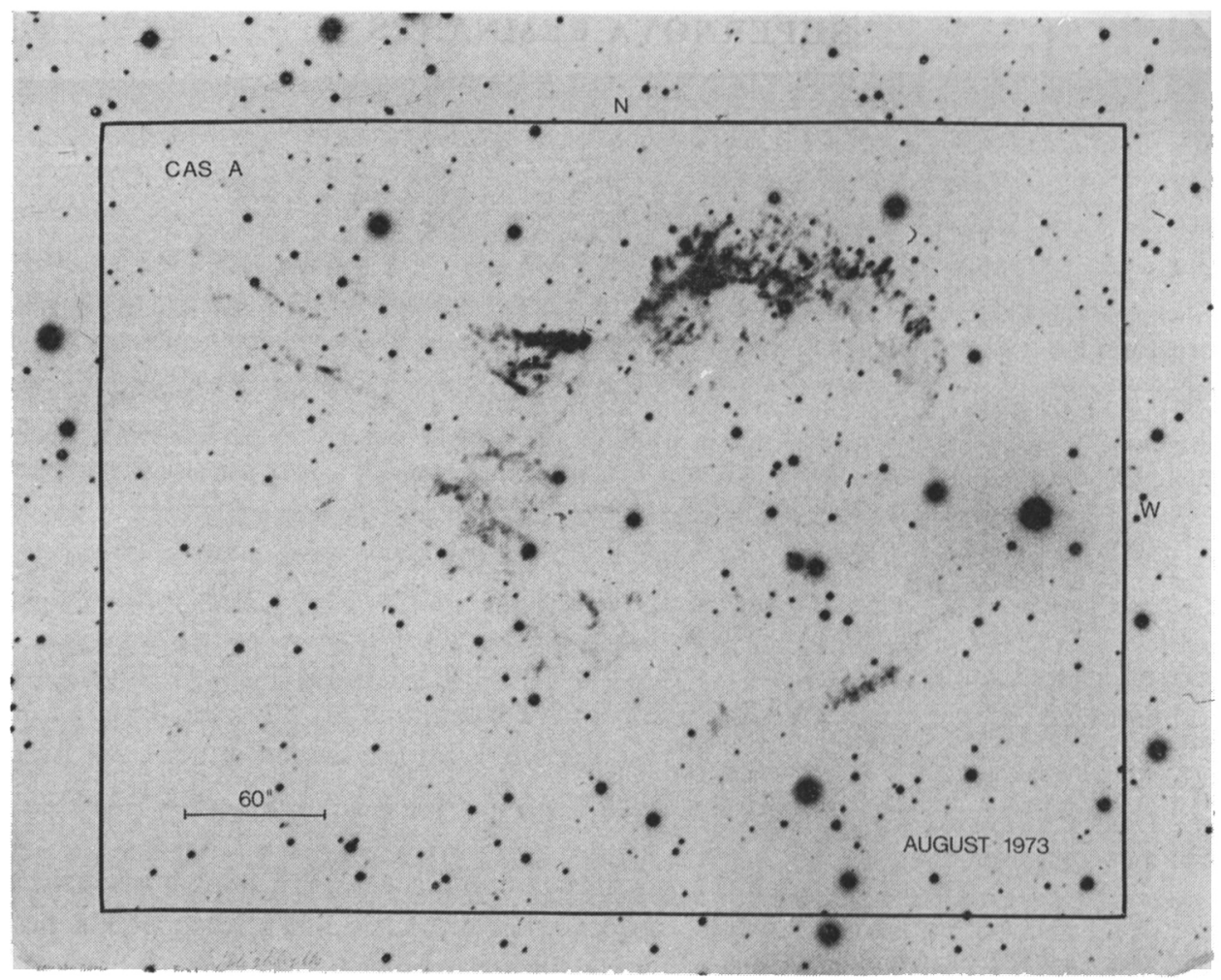

Fig. 1. 200-min exposure of Cas A with the Hale telescope through an [S II] interference filter.

C. The streak marked D appears to be a luminous but stationary conduit through which moving knots are seen to pass. Possibly this object resembles the streaks in the 'jet' that is located in the NE part of the remnant of Cas A.

(d) Nebulosity in which [O III] was brighter on a 120-min exposure than was [S II] on a 200 -min exposure is marked in Figure 3. This figure shows that the distribution of the knots that are particularly bright in [O III] $\lambda \lambda 4959,5007$ differs radically from that of the other moving nebulosity. In the northern part of Cas A this [O III] nebulosity seems to outline a well defined filament. In the eastern part of this filament individual knots are invisible on a deep 098-02 + RG2 ( $\lambda \lambda 6300-6900)$ exposure. The western part of the same filament is easily visible on this same plate but is invisible on a deep interference filter plate covering the wavelength range $\lambda \lambda 6650-6830$. This suggests that the western part of this filament probably radiates strongly in the range $\lambda \lambda 6300-6650$. Since no other moving knots have yet been observed to show $\mathrm{H} \alpha$ or [N II] it follows that [O I] $\lambda \lambda 6300,6364$ is a logical candidate for the emission in these knots. Nevertheless it seems remarkable that a knot should emit [O I] and [O III] but not [S II]. Clearly it would be very desirable to obtain spectra of these knots. 


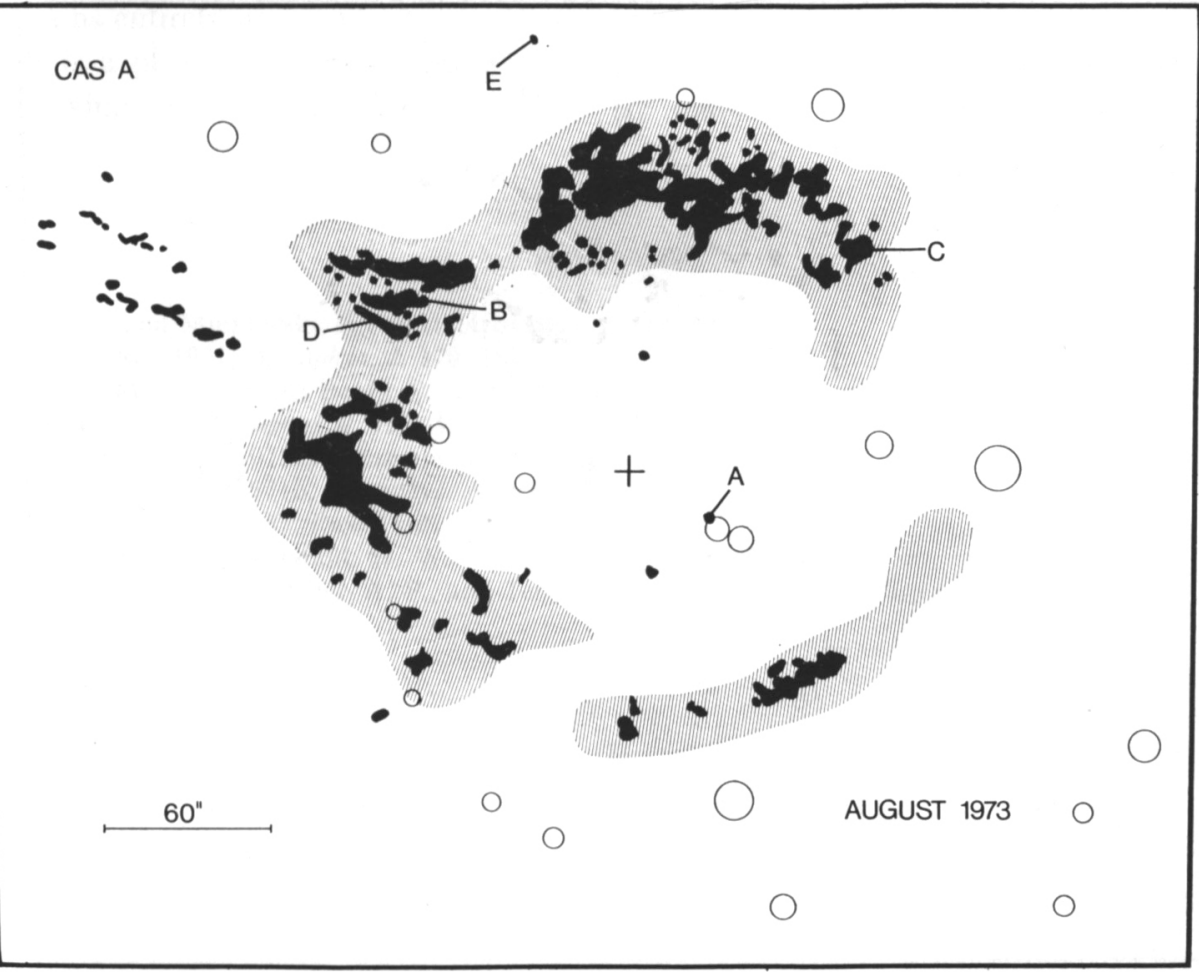

Fig. 2. Distribution of nebulosity in which $[\mathrm{S} \mathrm{II}]$ is strong relative to [O III]. No [O III] is visible in the knots labelled A, B and C. The centre of expansion of Cas A (van den Bergh and Dodd, 1970 ) is marked by a cross.

Inspection of Figure 3 suggests that the filament of nebulosity in which [O III] is particularly strong might be physically connected with filament No. 1 of Baade and Minkowski.

(e) The knot marked $\mathrm{E}$ in Figure 2 was clearly visible on the first red plate of Cas A that Walter Baade took 22 years ago. During the intervening period this isolated knot has travelled $\sim 10^{\prime \prime}$, i.e. approximately ten times its own diameter. This observation suggests that such fast-moving knots are produced by dense blobs of matter that are ploughing through a more or less stationary interstellar (or circumstellar) medium. The anomalous composition of these blobs (Peimbert and van den Bergh, 1971; Peimbert, 1971) shows that they were ejected from the Cas A supernova.

\section{Changes in Cas $A$}

Figure 1 shows the appearance of Cas A in August of 1973. Comparison of this plate with plates taken 20 years ago (see Figure 2 of Baade and Minkowski, 1954) shows the following: 
CAS A

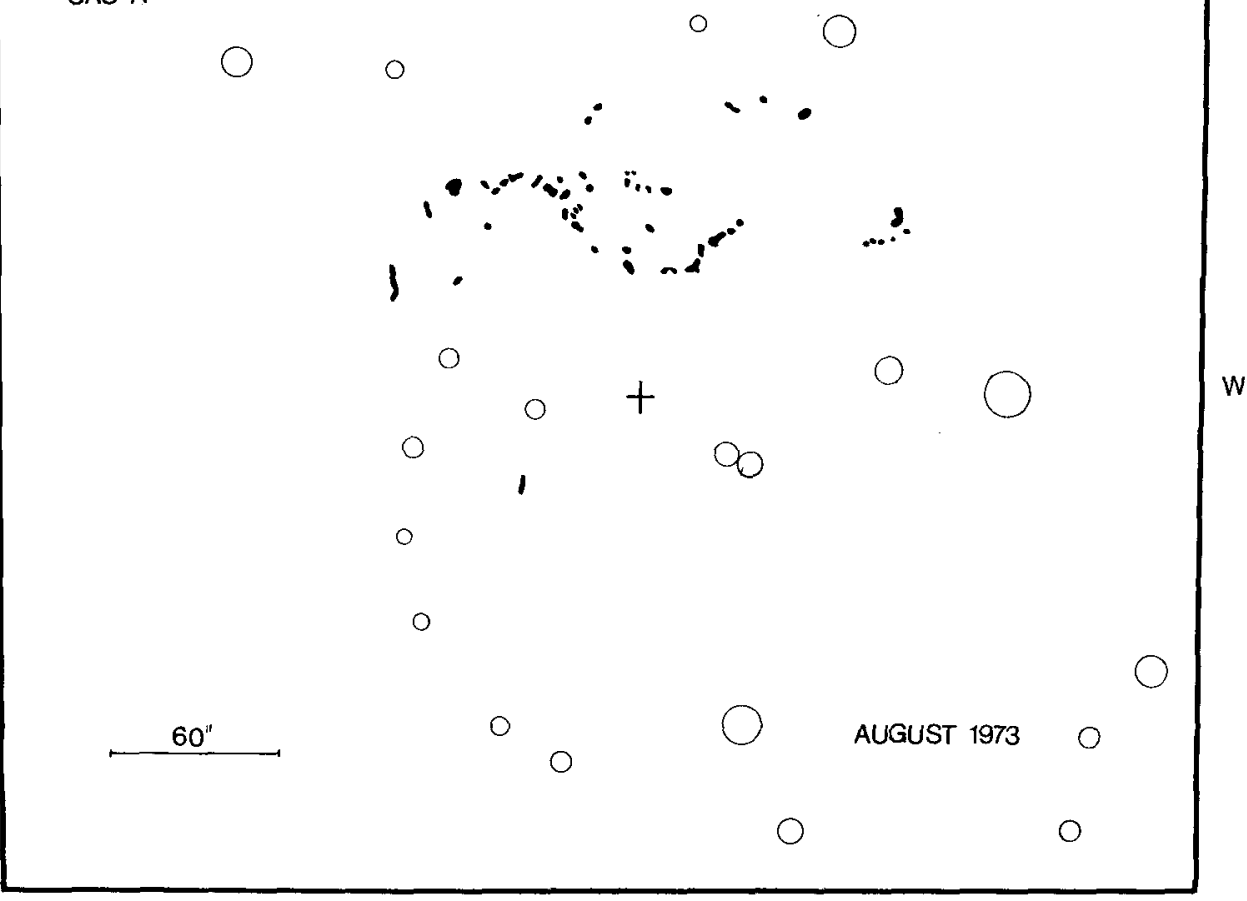

Fig. 3. Distribution of knots in which [O III] is particularly strong. Note that most of this nebulosity appears to be distributed in a single filament.

(a) A number of bright knots have recently developed along the southern rim of this supernova remnant. The intensity ratio of [S II] to [O III] in these new knots is similar to that observed in the majority of moving knots. A spectrum of knots $11 \mathrm{a}-11 \mathrm{~d}$ (see van den Bergh, 1971b) shows that the internal velocity dispersion in these new knots is similar to that observed in moving knots in other parts of the remnant.

(b) Typical moving knots have lifetimes $\sim 10 \mathrm{yr}$. Only a few of the brightest knots have remained visible during the entire 22 year time space covered by available 200-in. plates. Small faint knots typically have lifetimes of only a few years.

(c) The overall position of the bright arc of nebulosity situated to the NNW of the centre of expansion has not changed during the last 20 years despite the fact that individual knots in this arc have moved $\sim 8^{\prime \prime}$. This observation suggests that moving knots in this region light up as they pass through a stationary interstellar (or circumstellar) cloud bank.

(d) The brightest filament in the NE part of the remnant (Baade and Minkowski's No. 1) is gradually breaking up into individual knots. Radial velocity observations (van den Bergh, 1971b) show that individual knots in this filament have velocities 
that differ by up to $\sim 3000 \mathrm{~km} \mathrm{~s}^{-1}$. The origin of these large velocity differences remains entirely obscure.

It is a pleasure to thank the Hale Observatories for their generosity in making observing time available during the last six years for the continued study of the evolution of this enigmatic object.

\section{References}

Baade, W. and Minkowski, R.: 1954, Astrophys. J. 119, 206.

Peimbert, M.: 1971, Astrophys. J. 170, 261.

Peimbert, M. and van den Bergh, S.: 1971, Astrophys. J. 170, 261.

Searle, L.: 1971, Astrophys. J. 168, 41.

Van den Bergh, S.: 1971a, Astrophys. J. 165, 259.

Van den Bergh, S.: 1971b, Astrophys. J. 165, 457.

Van den Bergh, S.: 1973, Publ. Astron. Soc. Pacific 85, 335.

Van den Bergh, S. and Dodd, W. W.: 1970, Astrophys. J. 162, 485.

Van den Bergh, S., Marscher, A. P., and Terzian, Y.: 1973, Astrophys. J. Suppl. 26, 19 (No. 227). 